\title{
Grouping of clusters for efficient data aggregation in heterogeneous sensor networks for Cyber Physical System
}

\author{
Umapathi G $\mathbf{R}^{1}$,Dr. Ramesh Babu $\mathbf{H} \mathbf{S}^{2}$ \\ ${ }^{1}$ Department of Information Science and Engineering, Acharya Institute of Technology, Bangalore, India \\ ${ }^{2}$ Sai Vidya Institute of Technology, Bangalore, India
}

Received Date : January 01, 2022 Accepted Date : January 27, 2022 Published Date : February 07, 2022

\begin{abstract}
Wireless sensor networks (WSNs) consist of many sensor nodes. WSN are widely used in applications like habitat monitoring, disaster management, security and military, etc. Wireless sensor nodes have limited processing capability with very low battery power. This restriction of low battery power does not allow the sensor nodes to be alive for longer time. Therefore energy conservation has to take place. In this context Data aggregation technique is used. It is the process of aggregating of data and sending to the base station. Hence it reduces the number of packets to be sent to sink. Data aggregation technique increases the network lifetime by reducing the energy consumption.
\end{abstract}

Key words: $\mathrm{CH}$,clusters, $\mathrm{LEACH}$,sink, WSN,CPS

\section{INTRODUCTION}

Wireless sensor network is one of the emerging technologies for many real time applications. Wireless sensor network consists of nodes which have the capacity of sensing the environmental conditions like temperature, humidity, light. Each node is equipped with microprocessor, memory, transceiver and power supply. These nodes are deployed in physical area and connected through internet. Applications of wireless sensor network can be found in battlefield surveillance, industries, environmental monitoring [1]. Sensor nodes can be easily added and removed from the network. Thus provides scalability and flexibility.

In terms of energy efficiency and load balancing traditional routing protocols for WSN are not enough optimal [2]. Therefore Clustering was introduced for load balancing and to increase network lifetime. Clustering refers to the process of grouping of nodes to form a cluster. A network may consist of several clusters. In each cluster there is a leader node. It is also called as cluster head $(\mathrm{CH})$. $\mathrm{CH}$ takes data from all the nodes in its cluster. Cluster head aggregate all the data received from cluster members and then sends that data to the base station. The transmission between cluster members and cluster head is said to be intra cluster communication, whereas the transmission between cluster head and sink is known as inter cluster communication.

The three approaches for clustering process are centralized, distributed and hybrid [2]. In centralized clustering, the clusters and cluster heads are made by a centralized authority. All the nodes in the clusters can take the decision of becoming cluster head for the current round in distributed clustering. The mixture of both of above is Hybrid clustering. In clustering process, following properties are used in the structure of the cluster [3].

- Cluster Count

- Cluster Size

- Cluster density

- Message count

- Stability

- Intra-cluster Topology

- Inter-cluster head connectivity

The factors that should be taken care during designing a protocol or an algorithm for the sensor networks are described below [3].

- Fault Tolerance

- Scalability

- Production Costs

- Hardware Constraints

- Power Consumption

- Network Topology 


\section{RELATED WORK}

2.1Hierarchical networks

Flat networks can burden computation at the sink which leads to excessive usage of energy. If sink runs out of energy the whole network fails to operate. Thus to improve scalability and energy efficiency many hierarchical data aggregation approaches have been proposed.

2.1.1 LEACH (Low energy adaptive clustering hierarchy)

In WSN LEACH is one of the most popular algorithm [4].Here the sensor nodes are grouped together to form cluster. In LEACH a node with highest energy is elected as $\mathrm{CH}$ randomly in each rotation. First is the setup phase. It involves the grouping of nodes into clusters and selecting the cluster heads. Second phase is the steady state phase which involves data aggregation and data transmission.

2.1.2 LEACH-A (Advanced Low energy adaptive clustering hierarchy)

It is a heterogeneous energy protocol that helps to reduce the probability of nodes failure and to extend the time period before the first node dies which is called as stability period. Each sensor node keeps track of starting times of each round using a synchronized clock. A node with more energy is elected as $\mathrm{CH}$ for each cluster and these nodes are called as CAG node [5].

2.1.3LEACH-B (Balanced Low-energy Adaptive Clustering Hierarchy)

It uses decentralized algorithms [6].Here each node knows only its own position and destination node position. Here cluster formation and data transmission is done with the help of multiple accesses to different nodes. Energy dissipated along the path from originating node and destination node is calculated and based on this $\mathrm{CH}$ is elected.

\subsubsection{C-LEACH (Cell Low-energy Adaptive Clustering Hierarchy) \\ Here the network is divided into One sensor node in each cell is chosen as Cell Head. Several cells are grouped together to form clusters and each cluster has Cluster Head. On the basis of TDMA technique, each cell head will allocate a time to each sensor nodes to send the data to the cell head and cluster head [7].}

2.1.5 I-LEACH (Improved Low-energy Adaptive Clustering Hierarchy)

This protocol considers remaining energy in each node. Since each node has different energy selection of $\mathrm{CH}$ can be done efficiently. Each node in the cluster has a $\mathrm{CH}$ in its neighborhood. ILEACH addresses the uniform distribution of $\mathrm{CH}$ [ 8]

2.1.6.LEACH-S(Solar aware centralized and Distributed Low-energy Adaptive Clustering Hierarchy)

In centralized LEACH-S based on improved central control algorithm the sink selects the $\mathrm{CH}$. Along with the energy of the nodes, solar status is taken into account. The node with high energy is selected as $\mathrm{CH}$. solar aware nodes are given more importance than the battery driven nodes to select CHs [9]

2.1.7HEED(Hybrid energy efficient distributed clustering approach)

HEED extends the basic method of LEACH by using residual energy and angle between the nodes as a parameter for selection of cluster head[10]. An initial set of Cluster Heads are selected based on the residual energy while the secondary parameter is used for breaking ties.

2.1.5 TEEN(Threshold Sensitive Energy Efficient Sensor Network Protocol)

The main features of this protocol is that data which are time critical reaches almost instantaneously to the users depending upon the criticality of the sensed data attribute and the target application the soft threshold can be varied and more accurate picture of the network can be obtained when the soft threshold value is small[11].

2.1.6 APTEEN Threshold Sensitive Energy Efficient Sensor Network Protocol

APTEEN includes the functionality of both LEACH and TEEN. It captures the data periodically and also reacts to events which are time-critical [12]. If there is any sudden change in the value of the sensed data attribute, it reacts by sending report to the corresponding changed values to their Cluster heads.

2.1.7 SEP(Stable Election Protocol)

SEP is a heterogeneous-aware protocol $[13,14]$ to extend the time interval before the death of the first node. This is very important for many applications 
where the feedback from the sensor network must be reliable. SEP is based on weighted election probabilities of each node. Based on remaining energy in each node, the cluster head is elected. The advanced node and normal nodes have different residual energy.

\subsection{Chain based data aggregation}

In cluster based networks, all nodes send their data to cluster head and it aggregates all the data. If the cluster head is far away from the sink then more energy is required for data transmission. The key idea is that each sensor node transmits only to its closest neighbor.

2.2.1 PEGASIS(Power efficient data gathering protocol for sensor information systems)

The chain construction is performed in a greedy way. In the phase of data fusion sensor node sends data to its closest neighbor. To locate the closest neighbor, each node uses its signal strength to measure the distance to all neighboring nodes and then adjusts the strength of the signal so that only one node can be heard. When a node fails or dies due to low battery power, the chain is reconstructed using the same greedy approach by eliminating the failed sensor [15].

\subsubsection{EB-PEGASIS(Energy Balancing PEGASIS)}

A node will consider average distance of formed chain. It calculates the distance from its closest node and its upstream node .If the distance is more than distance-threshold, and then the closest node is a far. If the closest node joins the chain; it will form a long chain. In this situation, the far node will search a nearer node on formed chain [16].

\subsection{Grid based data aggregation}

Here data aggregation is performed by dividing the region monitored by the sensor nodes into several grid [17].They are grid based data aggregation and in-network based data aggregation. In grid based data aggregation, set of sensor nodes is assigned as data aggregators in fixed region of sensor network. The nodes send data directly to data aggregator of that grid. But nodes within that grid do not communicate with each other.

In-network based data aggregation, there are two main differences. They are each nodes within grid communicates with its neighboring nodes and any node within a grid can act as data aggregator. Here each node sends its signal strength to its neighbors. If the neighboring nodes have higher signal strength, sender stops sending packets. After all packets are received from all neighbors, the node with highest signal strength becomes the data aggregator.

\subsection{Tree based data aggregation}

Here sensor nodes are organized into tree like structure where data aggregation is performed at the leaf nodes and fused data is sent to the root node. Suitable for in-network data aggregation. Main aspect of the tree based data aggregation is the construction of an energy efficient data aggregation tree[18].

2.4.1 EADAT(Energy aware distributed approach) Sink initiates by broadcasting control messages. Sink acts as the root nodes. Control message has five fields: ID, parent, power, status and hop count. EADAT maximizes network lifetime and conserve energy [2].

2.4.2 PEDAP(power efficient data gathering and aggregation protocol)

PEDAP is based on a minimum spanning tree which improves the network lifetime even when the sink is inside the network field. When the base station is inside the sensor field by computing a minimum spanning tree over the sensor network with link costs, minimum energy is spent in each round [1].

\section{PROPOSED WORK}

The GCEDA algorithm operates in three phases .They are

1).Cluster formation

2).Intra-cluster aggregation

3). Inter-cluster aggregation

In the initial phase, the sensor nodes are organized into the group to form clusters. According to highest energy, minimum distance to sink cluster head is selected for each cluster. In the second phase, Cluster Head is responsible for aggregation of data packets generated by the source nodes.In intra-cluster aggregation, all sensor nodes send their sensed data to their Cluster Heads and this cluster head collects these data packets. By using aggregation functions cluster head performs the aggregation of data packets. Here the communication takes place inside the cluster between cluster members and cluster head. This phase is repeated in every cluster within the network. In the third phase, clusters are grouped to perform the further aggregation for transmission to the sink. Grouping of nodes in intra-cluster and 
grouping of clusters at inter cluster reduces the data packet count at the sink. Finally the sink receives the aggregated packet. The flow of the algorithm is shown in fig 1 . In this way the GCEDA algorithm works. This effectively reduces the energy consumption and increases the network lifetime [19].
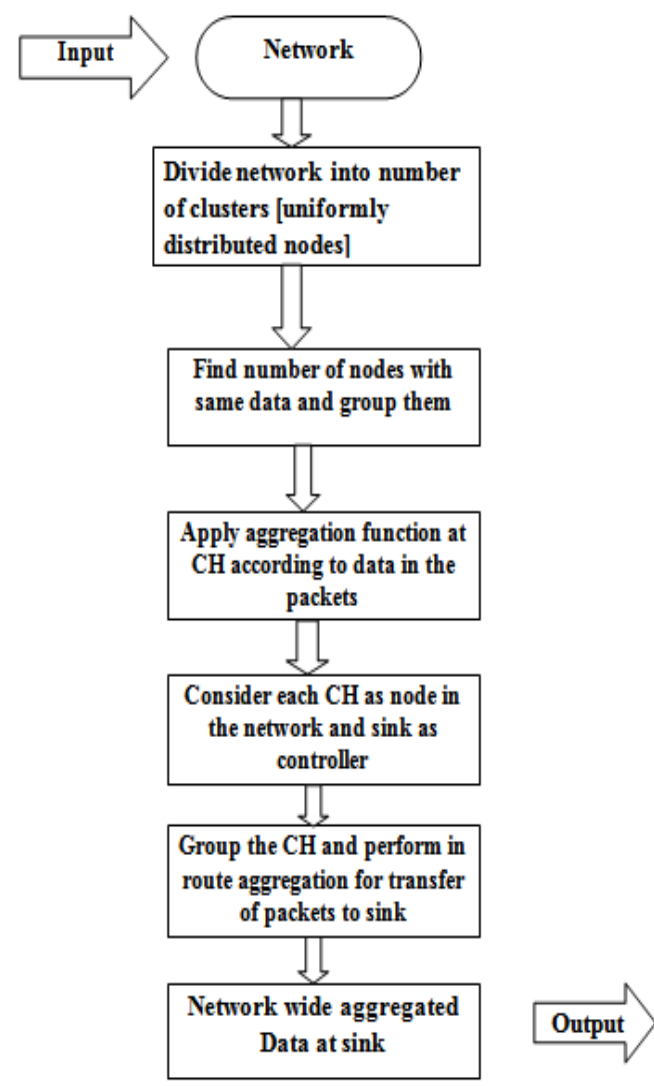

Figure 1: Flow of algorithm

In our proposed system, we have used different energy levels for each group.Group-1 has nodes which has energy level below 1200J.Group-2 nodes have energy level between 1200J and 1600J.Group-3 has nodes whose energy level is greater than 1600J.Thus addition of heterogeneity in the network improves the stability

\section{SIMULATION SET UP}

Table 1: Simulation Parameters

\begin{tabular}{|l|l|}
\hline Channel Type & Channel/Wireless channel \\
\hline $\begin{array}{l}\text { Radio-Propagation } \\
\text { model }\end{array}$ & $\begin{array}{l}\text { Propagation/Two Ray } \\
\text { Ground }\end{array}$ \\
\hline Network interface type & Phy/Wireless Phy \\
\hline MAC type & MAC/802.11 \\
\hline Interface queue type & Queue/Drop Tail/Pri Queue \\
\hline Link layer type & LL \\
\hline Antenna model & Antenna/Omni Antenna \\
\hline \multicolumn{1}{|c|}{ Max packet in freq } & 50 \\
\hline Number of mobile & 69 \\
\hline
\end{tabular}

\begin{tabular}{|l|l|}
\hline nodes & \\
\hline Routing protocol & DSDV \\
\hline $\begin{array}{l}\text { X dimension of } \\
\text { topography }\end{array}$ & 1101 \\
\hline $\begin{array}{l}\text { Y dimension of } \\
\text { topography }\end{array}$ & 1000 \\
\hline Time of simulation end & 50 \\
\hline
\end{tabular}

\section{RESULTS}

Here the proposed method is implemented in NS-2 and is compared with the system where there is no grouping of clusters at the network level.

\subsection{Performance Evaluation of Proposed} Mechanism with respect to control overhead in fig2

When there is more number of nodes the control overhead also increases. This results in frequent breaks in the links due to increase in the number of nodes. When the link is broken, the node transmits more control packets to source so that the control overhead is increased. Control Overhead is measured as the number/size of routing control packets transmitted by the protocol. It may be expressed as a ratio of control to data.

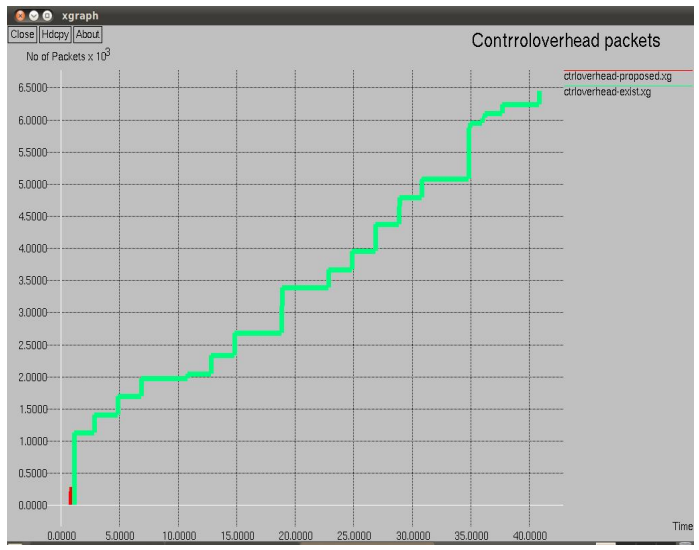

Figure 2: Control overhead vs. Time

\subsection{Performance Evaluation of Proposed} Mechanism with respect to bit error rate in fig3

A device which is used to measure the BER for a given transmission is called as BERT (bit error rate test or tester). The bit error rate (BER) is calculated by comparing the transmitted sequence of bits that have errors with respect to the total number of bits received. The BER indicates how often a data packet needs to be resent because of an error. BER is affected by many factors such as signal to noise, distortion and jitter. Lowering the data rate may reduce the BER. 


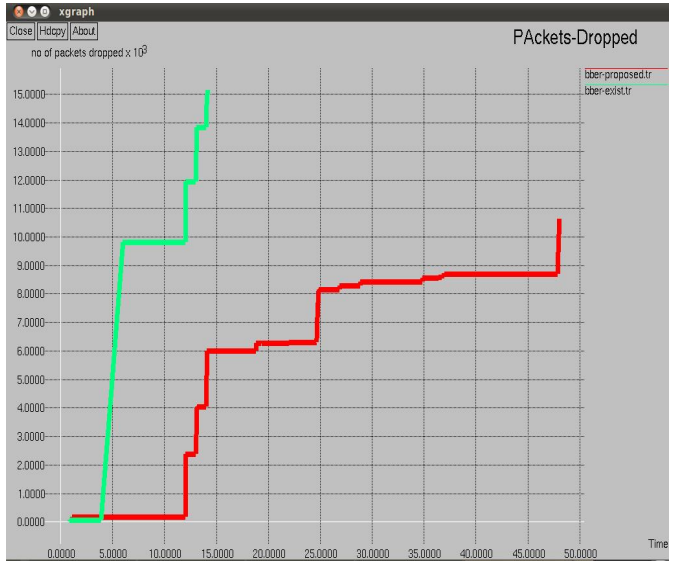

Figure 3: Packet dropped vs. Time

\subsection{Performance Evaluation of Proposed Mechanism with respect to packet delivery ratio in fig4}

Packet delivery fraction is calculated by measuring the percentage of total number of data packets received out of total number of data packets sent. Packet delivery ratio is an important aspect which describes the how much effective a routing protocol is. Data packets may get lost sometimes because of broken path or congestion along the route.

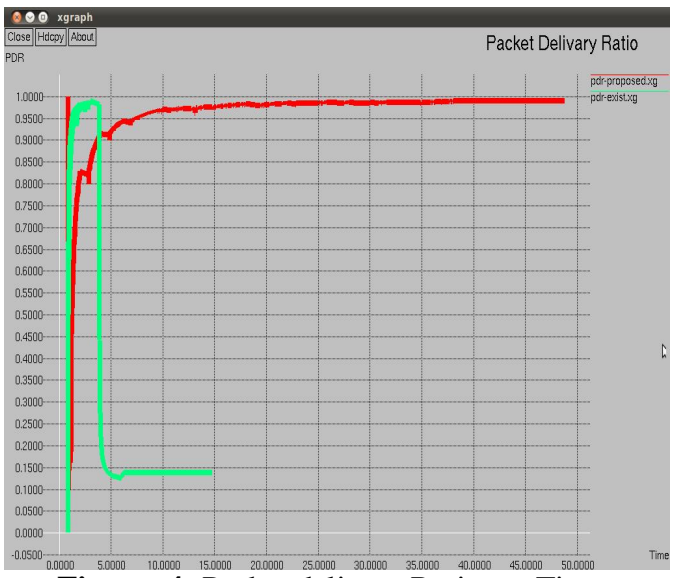

Figure 4: Packet delivery Ratio vs. Time

\subsection{Performance Evaluation of Proposed Mechanism with respect to throughput in fig 5}

Throughput is the amount of work that a system can do in a given period of time. Here throughput of the proposed method is compared with throughput of existing method where there is no grouping of clusters.

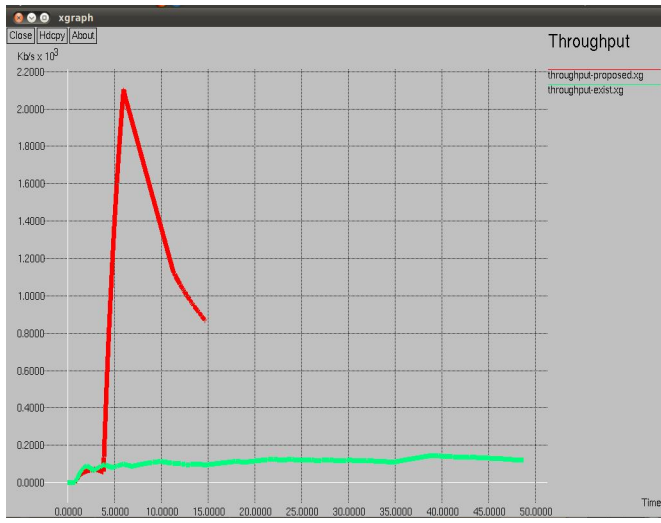

Figure 5: Throughput vs. Time

\section{CONCLUSION}

In last few years, in wireless sensor networks energy conservation has become one of the most important research areas. In this work, we proposed a method for grouping of clusters for efficient data aggregation for heterogeneous wireless sensor network. Analysis and simulations were conducted to evaluate the performance of the proposed method. The results show that the grouping of clusters method provides better performance when compared to method where no grouping of clusters is done. The addition of heterogeneity helped to increase the lifetime of network. The results also show that there will be more throughput.

Future work can be focused on reducing the number of cluster heads and also we can concentrate about providing the security mechanisms to protect data when nodes are sending the data to their cluster heads.

\section{REFERENCES}

[1]Rajagopalan, Ramesh and Varshney, Pramod $\mathrm{K}$,"Data aggregation techniques in sensor networks: A survey",Electrical Engineering and Computer Science, 2006

[2]Vaibhav Pandey, Amarjeet Kaur and Narottam Chand," A review on data aggregation techniques in wireless sensor Network", Journal of Electronic and Electrical Engineering ISSN: 0976-8106 \& E-ISSN: 0976-8114, Vol. 1, Issue 2, 2010.

[3]Naveen Sharma and Anand Nayyar ,"A Comprehensive Review of Cluster Based Energy Efficient Routing Protocols for Wireless Sensor Networks", IJAIEM, Volume 3, Issue 1, January 2014. 
[4]Soumitra Das,Pramod Ganjewa, 'Energy Efficient Cluster based Hierarchical Routing Protocols in Wireless Sensor Network - a Survey", MJRET, Volume 1, Issue 1, Pg. 06-22, April 2014.

[5]Amit Bhattacharjee, Balagopal Bhallamudi and Zahid Maqbool, "Energy-Efficient Hierarchical Cluster Based Routing Algorithm in Wsn: A Survey", in International Journal of Engineering Research \& Technology (IJERT), Vol. 2 Issue 5, May, 2013

[6] J.Gnanambigai, Dr.N.Rengarajan, .Anbukkarasi, "Leach and Its Descendant Protocols: A Survey", in International Journal of Communication and Computer Technologies, Vol. 01, No.3, Issue 02, September 2012

[7]Ravneet Kaur,Deepika Sharma and Navdeep Kaur, "Comparative Analysis Of Leach And Its Descendant Protocols In Wireless Sensor Network", International Journal of P2P Network Trends and TechnologyVolume3,Issue1,2013

[8]Naveen Kumar Jasbir Kaur,'Improved LEACH Protocol for Wireless Sensor Networks",IEEE,2011

[9]M. Aslam,N. Javaid, A. Rahim, U. Nazir, A. Bibi, Z. A. Khan," Survey of Extended LEACH-Based Clustering Routing Protocols for Wireless Sensor Networks", 11 Jul 2012

[10]Khushboo Pawar, Y.Kelkar,"A Survey of Hierarchical Routing Protocols in Wireless Sensor Network", International Journal of Engineering and Innovative Technology (IJEIT) Volume 1, Issue 5, May 2012

[11]Neetika and Simarpreet Kaur," Review on hierarchical routing in wireless sensor networks", International Journal of Smart Sensors and Ad Hoc Networks (IJSSAN), Vol-2, Iss-3,4, 2012

[12]V.Chandrasekaran, Dr.A.Shanmugam,"A review on hierarchical cluster based routing in wireless sensor networks ",jgrcs, Volume 3, No. 2, February 2012

[13]Yogesh Kumar,Kanwaljit Singh," Enhanced IB- LEACH for Heterogeneous Wireless Sensor Networks",IJNS, Volume 1, No.1, July August- September 2012.

[14]C.Divya, N.Krishnan, T.Gandhi mathy,"Energy Efficient Stable Election Protocol for Clustered Heterogeneous
Wireless Sensor Networks", (IOSRJCE),Volume 12, Issue 5 ,Jul. - Aug. 2013

[15]Parminder Kaur,Mrs.Mamta Katiyar," The Energy-Efficient Hierarchical Routing Protocols for WSN: A Review",ijarcsse, Volume 2, Issue 11, November 2012

[16]Shio Kumar Singh, M P Singh, D K Singh,"A Survey of Energy-Efficient Hierarchical Cluster-Based Routing in Wireless Sensor Networks",Int. J. of Advanced Networking and Applications, Volume: 02, Issue: 02,2010

[17]Sushruta Mishra, Hiren Thakkar," Features of WSN and Data Aggregation techniques in WSN: A Survey", IJEIT,Volume 1, Issue 4, April 2012

[18]Liyang Yu, Neng Wang, Wei Zhang,Chunlei Zheng,"Deploying a Heterogeneous Wireless Sensor Network",IEEE,2007

[19]Dnyaneshwar Mantri Neeli R Prasad Ramjee Prasad," Grouping of Clusters for Efficient Data Aggregation (GCEDA) in Wireless Sensor Network", 3rd IEEE International Advance Computing Conference (IACC), 2013. 